\title{
Microlensing Binaries with Candidate Brown Dwarf Companions
}

Shin, I.-G; Han, C.; Gould, A.; Udalski, A.; Sumi, T.; Dominik, M.; Beaulieu, J.-P; Tsapras, Y.; Bozza, V.; Szyman'ski, M. K.

Total number of authors:

155

Published in:

Astrophysical Journal

Link to article, DOI:

$10.1088 / 0004-637 X / 760 / 2 / 116$

Publication date:

2012

Document Version

Publisher's PDF, also known as Version of record

Link back to DTU Orbit

Citation (APA):

Shin, I. -G., Han, C., Gould, A., Udalski, A., Sumi, T., Dominik, M., Beaulieu, J. -P., Tsapras, Y., Bozza, V., Szyman'ski, M. K., Kubiak, M., Soszyn'ski, I., Pietrzyn'ski, G., Poleski, R., Ulaczyk, K., Pietrukowicz, P., Kozowski, S., Skowron, J., Wyrzykowski, ., ... Zub, M. (2012). Microlensing Binaries with Candidate Brown Dwarf Companions. Astrophysical Journal, 760:116(2), 1-10. https://doi.org/10.1088/0004-637X/760/2/116

\section{General rights}

Copyright and moral rights for the publications made accessible in the public portal are retained by the authors and/or other copyright owners and it is a condition of accessing publications that users recognise and abide by the legal requirements associated with these rights.

- Users may download and print one copy of any publication from the public portal for the purpose of private study or research.

- You may not further distribute the material or use it for any profit-making activity or commercial gain

- You may freely distribute the URL identifying the publication in the public portal 


\title{
MICROLENSING BINARIES WITH CANDIDATE BROWN DWARF COMPANIONS
}

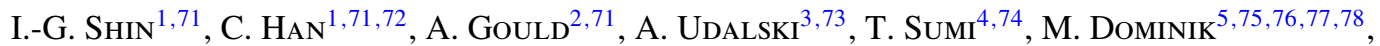 \\ J.-P. Beaulieu ${ }^{6,77}$, Y. Tsapras ${ }^{7,8,75}$, V. Bozza ${ }^{9,10,76}$, \\ AND
}

M. K. Szymański ${ }^{3}$, M. KubiaK $^{3}$, I. Soszyński ${ }^{3}$, G. Pietrzyński ${ }^{3,11}$, R. Poleski $^{3}$, K. UlaCzyK $^{3}$, P. Pietrukowicz $^{3}$,

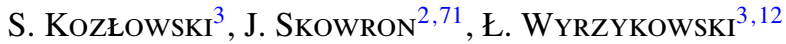

(The OGLE COLlaboration)

F. AbE ${ }^{13}$, D. P. Bennett ${ }^{14,77}$, I. A. Bond ${ }^{15}$, C. S. Botzler ${ }^{16}$, M. Freeman ${ }^{16}$, A. Fukui ${ }^{13}$, K. Furusawa ${ }^{13}$, F. Hayashi ${ }^{13}$, J. B. Hearnshaw ${ }^{17}$, S. Hosaka ${ }^{13}$, Y. Itow ${ }^{13}$, K. Kamiya ${ }^{13}$, P. M. Kilmartin ${ }^{18}$, S. Kobara ${ }^{13}$, A. Korpela ${ }^{19}$, W. LiN $^{15}$,

C. H. Ling ${ }^{15}$, S. Makita ${ }^{13}$, K. Masuda ${ }^{13}$, Y. Matsubara ${ }^{13}$, N. MiYaKe ${ }^{13}$, Y. Muraki ${ }^{20}$, M. Nagaya $^{13}$, K. Nishimoto $^{13}$, K. Ohnishi ${ }^{21}$, T. Okumura ${ }^{13}$, K. Omori ${ }^{13}$, Y. C. Perrott ${ }^{16}$, N. Rattenburi ${ }^{16}$, To. Saito ${ }^{22}$, L. Skuljan $^{15}$, D. J. Sullivan $^{19}$, D. Suzuki ${ }^{4}$, W. L. Sweatman ${ }^{15}$, P. J. Tristram ${ }^{18}$, K. Wada ${ }^{4}$, P. C. M. Yock $^{16}$

(The MOA COLlaboration)

AND

G. W. Christie ${ }^{23}$, D. L. Depoy ${ }^{24}$, S. Dong ${ }^{25}$, A. GAL-YAM ${ }^{26}$, B. S. GAudi ${ }^{2}$, L.-W. Hung ${ }^{27}$, J. JANCZAK $^{28}$, S. KASPI $^{29}$, D. MAOZ $^{29}$, J. McCormick ${ }^{30}$, D. McGregor ${ }^{2}$, D. Moorhouse ${ }^{31}$, J. A. Muñoz ${ }^{32}$, T. Natusch ${ }^{23}$, C. Nelson ${ }^{33}$, R. W. Pogge $^{2}$, T.-G. Tan $^{34}$, D. Polishook ${ }^{29}$, Y. Shvartzvald ${ }^{29}$, A. Shrorer ${ }^{7,35}$, G. Thornley ${ }^{31}$, U. Malamud ${ }^{29}$, J. C. YeE$^{2}$, AND J.-Y. Choi ${ }^{1}$, Y.-K. Jung ${ }^{1}$, H. PARK ${ }^{1}$, C.-U. LEE ${ }^{36}$, B.-G. PARK ${ }^{36}$, J.-R. KoO ${ }^{36}$

(The $\mu$ FUN COLlaboration)

AND

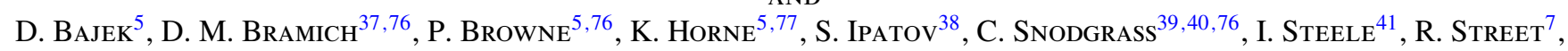
K. A. Alsubai ${ }^{38,75}$, M. J. Burgdorf ${ }^{42}$, S. Calchi Novati ${ }^{9,43}$, P. Dodds ${ }^{5,75}$, S. Dreizler ${ }^{44}$, X.-S. FAnG $^{45}$, F. Grundahl ${ }^{46}$, C.-H. Gu ${ }^{45}$, S. Hardis ${ }^{47}$, K. HarPs $\varnothing E^{47,48}$, T. C. Hinse ${ }^{36,47,49}$, M. HunderTMARK ${ }^{5,44,75}$, J. JeSSEN-HANSEN ${ }^{46}$,

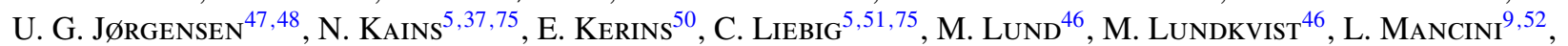
M. Mathiasen ${ }^{47}$, A. Hornstrup ${ }^{53}$, M. T. Penny ${ }^{50}$, S. Proft ${ }^{51}$, S. Rahvar ${ }^{54,8,55}$, D. Ricci ${ }^{56}$, G. Scarpetta $^{9,57}$, J. Skottfelt $^{47}$, J. Southworth ${ }^{58}$, J. Surdej ${ }^{56}$, J. Tregloan-Reed ${ }^{58}$, O. Wertz ${ }^{56}$, F. Zimmer ${ }^{51}$, M. D. Albrow ${ }^{17}$, V. Batista ${ }^{2,71}$,

S. Brillant ${ }^{40}$, J. A. R. Caldwell ${ }^{59}$, J. J. Calitz ${ }^{60}$, A. Cassan ${ }^{6}$, A. Cole $^{61}$, K. H. Cook ${ }^{62}$, E. Corrales ${ }^{6}$, Ch. Coutures ${ }^{6}$, S. Dieters ${ }^{6,63}$, D. Dominis Prester ${ }^{64}$, J. Donatowicz ${ }^{65}$, P. Fouqué ${ }^{63}$, J. Greenhill ${ }^{61}$, K. Hill ${ }^{61}$, M. HoffMan ${ }^{60}$, S. R. Kane ${ }^{66}$, D. Kubas ${ }^{6,40}$, J.-B. Marquette ${ }^{6}$, R. Martin ${ }^{67}$, P. Meintjes ${ }^{60}$, J. Menzies ${ }^{68}$, K. R. Pollard ${ }^{17}$, K. C. Sahu ${ }^{69,76}$,

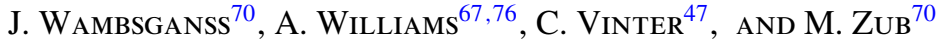

${ }^{1}$ Department of Physics, Institute for Astrophysics, Chungbuk National University, Cheongju 371-763, Republic of Korea

${ }^{2}$ Department of Astronomy, Ohio State University, 140 W. 18th Ave., Columbus, OH 43210, USA

${ }^{3}$ Warsaw University Observatory, Al. Ujazdowskie 4, 00-478 Warszawa, Poland

${ }^{4}$ Department of Earth and Space Science, Osaka University, Osaka 560-0043, Japan

${ }^{5}$ SUPA, University of St. Andrews, School of Physics \& Astronomy, North Haugh, St. Andrews KY16 9SS, UK

${ }^{6}$ Institut d'Astrophysique de Paris, UMR7095 CNRS-Université Pierre \& Marie Curie, 98 bis boulevard Arago, F-75014 Paris, France

${ }^{7}$ Las Cumbres Observatory Global Telescope Network, 6740B Cortona Dr., Suite 102, Goleta, CA 93117, USA

${ }^{8}$ School of Physics and Astronomy, Queen Mary, University of London, Mile End Road, London E1 4NS, UK

${ }^{9}$ Dipartimento di Fisica "E. R. Caianiello," Università degli Studi di Salerno, Via S. Allende, I-84081 Baronissi (SA), Italy

${ }^{10}$ Istituto Nazionale di Fisica Nucleare, Sezione di Napoli, Italy

${ }^{11}$ Departamento de Astronomia, Universidad de Concepción, Casilla 160-C, Concepción, Chile

${ }^{12}$ Institute of Astronomy, University of Cambridge, Madingley Road, Cambridge CB3 0HA, UK

${ }^{13}$ Solar-Terrestrial Environment Laboratory, Nagoya University, Nagoya 464-8601, Japan

${ }^{14}$ Department of Physics, University of Notre Dame, Notre Dame, IN 46556, USA

15 Institute of Information and Mathematical Sciences, Massey University, Private Bag 102-904, North Shore Mail Centre, Auckland, New Zealand

${ }^{16}$ Department of Physics, University of Auckland, Private Bag 92019, Auckland, New Zealand

${ }^{17}$ Department of Physics and Astronomy, University of Canterbury, Private Bag 4800, Christchurch 8020, New Zealand

${ }^{18}$ Mt. John Observatory, P.O. Box 56, Lake Tekapo 8770, New Zealand

${ }^{19}$ School of Chemical and Physical Sciences, Victoria University, Wellington, New Zealand

${ }^{20}$ Department of Physics, Konan University, Nishiokamoto 8-9-1, Kobe 658-8501, Japan

${ }^{21}$ Nagano National College of Technology, Nagano 381-8550, Japan

22 Tokyo Metropolitan College of Industrial Technology, Tokyo 116-8523, Japan

${ }^{23}$ Auckland Observatory, P.O. Box 24-180, Auckland, New Zealand

${ }^{24}$ Department of Physics, Texas A\&M University, College Station, TX 77843-4242, USA

${ }^{25}$ Institute for Advanced Study, Einstein Dr., Princeton, NJ 08540, USA

${ }^{26}$ Benoziyo Center for Astrophysics, The Weizmann Institute, Rehovot 76100, Israel

27 Department of Physics \& Astronomy, University of California, Los Angeles, CA 90095, USA

${ }^{28}$ Department of Physics, Ohio State University, 191 W. Woodruff, Columbus, OH 43210, USA

${ }^{29}$ School of Physics and Astronomy, Tel-Aviv University, Tel Aviv 69978, Israel

${ }^{30}$ Farm Cove Observatory, Pakuranga, Auckland

${ }^{31}$ Kumeu Observatory, Kumeu, New Zealand

${ }^{32}$ Departamento de Astronomiá y Astrofísica, Universidad de Valencia, E-46100 Burjassot, Valencia, Spain 


\author{
${ }^{33}$ College of Optical Sciences, University of Arizona, 1630 E. University Blvd., Tucson, AZ 85721, USA \\ ${ }^{34}$ Perth Exoplanet Survey Telescope, Perth, Australia \\ ${ }^{35}$ Department of Physics, University of California, Santa Barbara, CA 93106, USA \\ ${ }^{36}$ Korea Astronomy and Space Science Institute, 776 Daedukdae-ro, Yuseong-gu, Daejeon 305-348, Republic of Korea \\ ${ }^{37}$ European Southern Observatory, Karl-Schwarzschild-Strasse 2, D-85748 Garching bei München, Germany \\ ${ }^{38}$ Qatar Foundation, P.O. Box 5825, Doha, Qatar \\ ${ }^{39}$ Max-Planck-Institut für Sonnensystemforschung, Max-Planck-Strasse 2, D-37191 Katlenburg-Lindau, Germany \\ ${ }^{40}$ European Southern Observatory, Casilla 19001, Vitacura 19, Santiago, Chile \\ ${ }^{41}$ Astrophysics Research Institute, Liverpool John Moores University, Egerton Wharf, Birkenhead CH41 1LD, UK \\ ${ }^{42}$ HE Space Operations GmbH, Flughafenallee 24, D-28199 Bremen, Germany \\ ${ }^{43}$ Istituto Internazionale per gli Alti Studi Scientifici (IIASS), Vietri Sul Mare (SA), Italy \\ ${ }^{44}$ Institut für Astrophysik, Georg-August-Universität, Friedrich-Hund-Platz 1, D-37077 Göttingen, Germany \\ ${ }^{45}$ National Astronomical Observatories/Yunnan Observatory, Joint Laboratory for Optical Astronomy, Chinese Academy of Sciences, Kunming 650011, China \\ ${ }^{46}$ Department of Physics \& Astronomy, Aarhus Universitet, Ny Munkegade, DK-8000 Århus C, Denmark \\ ${ }^{47}$ Niels Bohr Institute, University of Copenhagen, Juliane Maries vej 30, DK-2100 Copenhagen, Denmark \\ ${ }^{48}$ Centre for Star and Planet Formation, Geological Museum, Øster Voldgade 5, DK-1350 Copenhagen, Denmark \\ ${ }^{49}$ Armagh Observatory, College Hill, Armagh BT61 9DG, UK \\ ${ }^{50}$ Jodrell Bank Centre for Astrophysics, University of Manchester, Oxford Road, Manchester M13 9PL, UK \\ ${ }^{51}$ Astronomisches Rechen-Institut, Zentrum für Astronomie der Universität Heidelberg (ZAH), Mönchhofstrasse 12-14, D-69120 Heidelberg, Germany \\ 52 Max Planck Institute for Astronomy, Königstuhl 17, D-69117 Heidelberg, Germany \\ ${ }^{53}$ Institut for Rumforskning og-teknologi, Danmarks Tekniske Universitet, Juliane Maries Vej 30, DK-2100 Copenhagen, Denmark \\ ${ }^{54}$ Department of Physics, Sharif University of Technology, P.O. Box 11155-9161, Tehran, Iran \\ ${ }^{55}$ Perimeter Institute for Theoretical Physics, 31 Caroline St. N, Waterloo, Ontario ON N2L 2Y5, Canada \\ ${ }^{56}$ Institut d'Astrophysique et de Géophysique, Allée du 6 Août 17, Sart Tilman, Bât. B5c, B-4000 Liège, Belgium \\ ${ }^{57}$ INFN, Gruppo Collegato di Salerno, Sezione di Napoli, Italy \\ ${ }^{58}$ Astrophysics Group, Keele University, Newcastle-under-Lyme ST5 5BG, UK \\ ${ }^{59}$ McDonald Observatory, 16120 State Hwy Spur 78 \#2, Fort Davis, TX 79334, USA \\ ${ }^{60}$ Faculty of Natural and Agricultural Sciences, Department of Physics, University of the Free State, P.O. Box 339, Bloemfontein 9300, South Africa \\ ${ }^{61}$ School of Math and Physics, University of Tasmania, Private Bag 37, GPO Hobart, Tasmania 7001, Australia \\ ${ }^{62}$ Institute of Geophysics and Planetary Physics (IGPP), L-413, Lawrence Livermore National Laboratory, P.O. Box 808, Livermore, CA 94451, USA \\ ${ }^{63}$ LATT, Université de Toulouse, CNRS, 14 Avenue Edouard Belin, F-31400 Toulouse, France \\ ${ }^{64}$ Faculty of Arts and Sciences, Physics Department, University of Rijeka, Omladinska 14, 51000 Rijeka, Croatia \\ ${ }^{65}$ Department of Computing, Technical University of Vienna, Wiedner Hauptstrasse 10, A-1040 Vienna, Austria \\ ${ }^{66}$ NASA Exoplanet Science Institute, Caltech, MS 100-22, 770 South Wilson Avenue, Pasadena, CA 91125, USA \\ ${ }^{67}$ Perth Observatory, Walnut Road, Bickley, Perth 6076, Australia \\ ${ }^{68}$ South African Astronomical Observatory, P.O. Box 9, Observatory 7935, South Africa \\ ${ }^{69}$ Space Telescope Science Institute, 3700 San Martin Dr., Baltimore, MD 21218, USA \\ ${ }^{70}$ Astronomisches Rechen-Institut (ARI), Zentrum für Astronomie der Universität Heidelberg (ZAH), Mönchhofstrasse 12-14, D-69120 Heidelberg, Germany \\ Received 2012 August 11; accepted 2012 October 3; published 2012 November 14
}

\begin{abstract}
Brown dwarfs are important objects because they may provide a missing link between stars and planets, two populations that have dramatically different formation histories. In this paper, we present the candidate binaries with brown dwarf companions that are found by analyzing binary microlensing events discovered during the 2004-2011 observation seasons. Based on the low mass ratio criterion of $q<$ 0.2, we found seven candidate events: OGLE-2004-BLG-035, OGLE-2004-BLG-039, OGLE-2007-BLG-006, OGLE-2007-BLG-399/MOA-2007-BLG-334, MOA-2011-BLG-104/OGLE-2011-BLG-0172, MOA-2011-BLG149, and MOA-201-BLG-278/OGLE-2011-BLG-012N. Among them, we are able to confirm that the companions of the lenses of MOA-2011-BLG-104/OGLE-2011-BLG-0172 and MOA-2011-BLG-149 are brown dwarfs by determining the mass of the lens based on the simultaneous measurement of the Einstein radius and the lens parallax. The measured masses of the brown dwarf companions are $0.02 \pm 0.01 M_{\odot}$ and $0.019 \pm 0.002 M_{\odot}$ for MOA-2011-BLG-104/OGLE-2011-BLG-0172 and MOA-2011-BLG-149, respectively, and both companions are orbiting low-mass $\mathrm{M}$ dwarf host stars. More microlensing brown dwarfs are expected to be detected as the number of lensing events with well-covered light curves increases with new-generation searches.
\end{abstract}

Key words: binaries: general - brown dwarfs - gravitational lensing: micro

Online-only material: color figures

\footnotetext{
71 The $\mu$ FUN Collaboration.

${ }_{72}$ Corresponding author.

${ }^{73}$ The OGLE Collaboration.

74 The MOA Collaboration.

75 The RoboNet Collaboration.

76 The MiNDSTEp Consortium.

77 The PLANET Collaboration.

${ }^{78}$ Royal Society University Research Fellow.
}

\section{INTRODUCTION}

Brown dwarfs are sub-stellar objects that are too low in mass to sustain hydrogen fusion reactions in their cores but much higher than planets. Studies of brown dwarfs are important because they may provide a missing link between stars and planets (Kulkarni 1997), two populations that have dramatically different formation histories. In addition, the Galaxy may be teeming with brown dwarfs, although there is no evidence for a large population of brown dwarfs from current observations 
(Graff \& Freese 1996; Najita et al. 2000; Tisserand et al. 2007). Brown dwarfs had long been thought to exist based on theoretical considerations (Kumar 1969). However, these objects are intrinsically faint, and thus the first confirmed brown dwarf was not discovered until 1995 (Rebolo et al. 1995; Nakajima 1995).

There have been two major methods of detecting brown dwarfs. The first is direct imaging by using ground-based (e.g., Schuh et al. 2003) and space-borne (e.g., Mainzer et al. 2011) infrared instruments. As in the case of extrasolar planets, brown dwarfs can also be indirectly discovered by detecting wobbles in the motion of the companion star or the flux decrease of the companion star caused by the brown dwarf occulting the companion star.

Microlensing is also an effective method to detect brown dwarfs. Gravitational microlensing refers to the astronomical phenomenon wherein the brightness of a star is magnified by the bending of light caused by the gravity of an intervening object (lens) between the background star (source) and an observer. Since the phenomenon occurs regardless of the lens brightness, microlensing was proposed to detect dark components of the Galaxy such as black holes, neutron stars, and brown dwarfs (Paczyński 1986).

Although effective, the application of the microlensing method in brown dwarf detections has been limited. The main reason for this is the difficulty in distinguishing brown dwarf events from those produced by main-sequence stars. For general lensing events in which a single mass causes the brightening of a background star, the magnification of the lensed star flux depends on the projected lens-source separation, $u$, by

$$
A=\frac{u^{2}+2}{u\left(u^{2}+4\right)^{1 / 2}},
$$

where the lens-source separation is normalized by the radius of the Einstein ring, $\theta_{\mathrm{E}}$ (Einstein radius). The lens-source separation is related to the lensing parameters of the timescale for the source to cross the Einstein radius, $t_{\mathrm{E}}$ (Einstein timescale), the time of the closest lens-source approach, $t_{0}$, and the lens-source separation at the moment of the closest approach, $u_{0}$ (impact parameter), by

$$
u=\left[\left(\frac{t-t_{0}}{t_{\mathrm{E}}}\right)^{2}+u_{0}^{2}\right]^{1 / 2}
$$

Among these lensing parameters, only the Einstein timescale provides information about the physical parameters of the lens. However, it results from the combination of the lens mass, distance, and transverse speed of the relative lens-source motion, and thus it is difficult to uniquely determine the mass of the lensing object. Not knowing the mass, then, it is difficult to single out brown dwarf events from those produced by stars. In principle, it is possible to measure the lens mass by additionally measuring the Einstein radius and the lens parallax, which are respectively related to the physical parameters of the lens by

$$
\theta_{\mathrm{E}}=\left(\kappa M \pi_{\mathrm{rel}}\right)^{1 / 2}
$$

and

$$
\pi_{\mathrm{E}}=\frac{\pi_{\mathrm{rel}}}{\theta_{\mathrm{E}}},
$$

where $\pi_{\text {rel }}=\operatorname{AU}\left(D_{\mathrm{L}}^{-1}-D_{\mathrm{S}}^{-1}\right), \kappa=4 G /\left(c^{2} \mathrm{AU}\right), M$ is the mass of the lens, and $D_{\mathrm{L}}$ and $D_{\mathrm{S}}$ are the distances to the lens and source star, respectively. The Einstein radius is measured by analyzing the distortion of the lensing light curve affected by the finite size of the source star (Nemiroff \& Wickramasinghe 1994; Witt \& Mao 1994; Gould 1994), while the lens parallax is measured by analyzing the deviation in a lensing light curve caused by the deviation of the relative lens-source motion from a rectilinear one due to the change of the observer's position induced by the orbital motion of the Earth around the Sun (Gould 1992). Unfortunately, the chance of simultaneously measuring $\theta_{\mathrm{E}}$ and $\pi_{\mathrm{E}}$ is very low for a single-lens event. The finite source effect is important only for very high magnification events, for which the lens-source separation is comparable to the source size (Choi et al. 2012), but these events are very rare. The parallax effect is important only for events with timescales that are a significant fraction of the orbital period of the Earth, i.e., 1 yr. However, such long-timescale events are unlikely to be produced by lowmass brown dwarfs because $t_{\mathrm{E}} \propto M^{1 / 2}$. As a result, there exist only two cases for which the lens of a single-lensing event was identified as a brown dwarf (Smith et al. 2003; Gould et al. 2009). ${ }^{79}$

In binary-lensing events, in which the lens is composed of two masses, on the other hand, the chance to identify the lens as a brown dwarf is relatively high. There are several reasons for this. First, for binary-lensing events, it is possible to routinely measure the mass ratio, $q$, of the lens components. Then, brown dwarf candidates can be sorted out based on the first-order criterion of small mass ratios. Second, the majority of binary-lensing events involve caustic crossings (Schneider \& Weiß 1986; Mao \& Paczyński 1991). The caustics represent the positions on the source plane at which the lensing magnification of a point source becomes infinite. The caustic-crossing part of a lensing light curve varies depending on the source size. Analysis of the light curve affected by this finite source effect allows one to measure the Einstein radius, which allows for better constraint on the lens mass. Third, the chance to measure the lens parallax is high, too. This is because the duration of an event corresponds to the total mass of the binary-lens system, not the low-mass component, and thus the average timescale of a binary-lens event is longer than that of a single-lens event.

Despite these advantages, there exists only one event (OGLE-2008-BLG-510) for which the companion of a binary lens is known to be a brown dwarf candidate (Bozza et al. 2012). The main reason for the rarity of brown dwarf events is the difficulty in analyzing binary-lensing light curves due to the complexity of the light curves. For binary-lensing events in which the mass ratio between the lens components is very low, as in the case of a binary composed of a star and a planet, the signature of the low-mass companion appears as a short-term deviation on the top of an otherwise smooth and symmetric single-lensing light curve of the primary. As a result, identification and analysis of planetary events are relatively simple, resulting in more than 20 identified microlensing planets. However, the perturbative nature of the companion breaks down and the light curve becomes very complex for binaries with mass ratios greater than $q \sim 0.1$, which corresponds to the mass ratio of a typical binary lens composed of a brown dwarf and a star. This introduces difficulties in the immediate identification of a brown dwarf event. A firm identification of a brown dwarf event requires detailed modeling of lensing light curves.

\footnotetext{
79 Among these two cases, the microlensing brown dwarf discovered by Gould et al. (2009) is the exception that provides the rule because the lens mass could be determined not by measuring the lens parallax induced by Earth's orbital motion but from the "terrestrial parallax."
} 
Table 1

Coordinates of Events

\begin{tabular}{|c|c|c|c|c|}
\hline Event & R.A. (J2000) & Decl. (J2000) & $l$ & $b$ \\
\hline OGLE-2004-BLG-035 & $17^{\mathrm{h}} 48^{\mathrm{m}} 43^{\mathrm{s}} .16$ & $-35^{\circ} 57^{\prime} 45^{\prime \prime} .9$ & 354.32 & -4.19 \\
\hline OGLE-2004-BLG-039 & $17^{\mathrm{h}} 53^{\mathrm{m}} 47^{\mathrm{s}} .58$ & $-30^{\circ} 52^{\prime} 11^{\prime \prime} .7$ & 359.25 & -2.51 \\
\hline OGLE-2007-BLG-006 & $18^{\mathrm{h}} 02^{\mathrm{m}} 52^{\mathrm{s}} .50$ & $-29^{\circ} 15^{\prime} 11^{\prime \prime} .8$ & 1.63 & -3.41 \\
\hline OGLE-2007-BLG-399/MOA-2007-BLG-334 & $17^{\mathrm{h}} 45^{\mathrm{m}} 35^{\mathrm{s}} .79$ & $-34^{\circ} 54^{\prime} 37^{\prime \prime} .5$ & 354.89 & -3.10 \\
\hline MOA-2011-BLG-104/OGLE-2011-BLG-0172 & $17^{\mathrm{h}} 54^{\mathrm{m}} 22^{\mathrm{s}} .48$ & $-29^{\circ} 50^{\prime} 01^{\prime \prime} .7$ & 0.21 & -2.10 \\
\hline MOA-2011-BLG-149 & $17^{\mathrm{h}} 56^{\mathrm{m}} 47^{\mathrm{s}} .69$ & $-31^{\circ} 16^{\prime} 04^{\prime \prime} .7$ & 359.23 & -3.27 \\
\hline MOA-2011-BLG-278/OGLE-2011-BLG-012N & $17^{\mathrm{h}} 54^{\mathrm{m}} 11^{\mathrm{s}} .32$ & $-30^{\circ} 05^{\prime} 21^{\prime \prime} .6$ & 359.97 & -2.19 \\
\hline
\end{tabular}

Unfortunately, modeling light curves of binary-lensing events is a difficult task due to the complexity of the $\chi^{2}$ surface in the parameter space combined with the heavy computation required for analysis. As a result, detailed modeling of binary events was conducted for a small subset of events, resulting in a small number of identified brown dwarfs. In recent years, however, there has been great progress in the analysis of binary events with the development of analysis methods based on advanced logic combined with improved computing power. This progress made the analysis not only more precise but also faster. As a result, nearly all binary-lensing events are routinely modeled in real time with the progress of events in current microlensing experiments (Ryu et al. 2010; Bozza et al. 2012).

In this paper, we present brown dwarf candidates found from the analyses of binary-lensing events detected from microlensing experiments conducted from 2004 to 2011. In Section 2, we describe the criteria of choosing brown dwarf candidates, observation of events, and data reduction. In Section 3, we describe the procedure of modeling. In Section 4, we present the results of the analysis. In Section 5, we draw our conclusions.

\section{SAMPLE AND OBSERVATIONS}

To search for brown dwarf candidates, we investigate binarylensing events discovered during the 2004-2011 microlensing observation seasons. Considering that typical Galactic microlensing events are produced by low-mass stars (Han \& Gould 2003 ), the lower mass companion of an event with a mass ratio $q<0.2$ is likely to be a brown dwarf candidate. Therefore, we sort out brown dwarf candidate events with the criterion of $q<0.2$. For this, we conduct modeling of all binary-lensing events with relatively good light-curve coverage combined with obvious binary-lensing features such as the spikes caused by crossings over the fold or approaches to the cusp of a caustic. The good coverage criterion is needed to secure the mass ratio measurement. The criterion of the characteristic features is needed because it is known that binary-lensing events with weak signals can often be interpreted as other types of anomalies such as binary sources (Jaroszyński \& Skowron 2008). For binary-lensing events detected before 2008, we also refer to the previous analyses conducted by Jaroszyński et al. (2006, 2010) and Skowron et al. (2007). From the 2004-2011 search, we found seven candidate events: OGLE-2004-BLG035, OGLE-2004-BLG-039, OGLE-2007-BLG-006, OGLE2007-BLG-399/MOA-2007-BLG-334, MOA-2011-BLG-104/ OGLE-2011-BLG-0172, MOA-2011-BLG-149, and MOA201-BLG-278/OGLE-2011-BLG-012N.

In Table 1, we list the candidate events that are analyzed in this work along with the equatorial and Galactic coordinates of the lensed stars. The name of each lensing event is formed by taking the survey group that discovered the event (i.e., OGLE or MOA), followed by the year of discovery (e.g., 2004), the direction of the observation field ("BLG" designating the Galactic bulge
Table 2

Telescopes

\begin{tabular}{|c|c|}
\hline Event & Telescopes \\
\hline OGLE-2004-BLG-035 & OGLE, $1.3 \mathrm{~m}$ Warsaw, Las Campanas, Chile \\
\hline OGLE-2004-BLG-039 & OGLE, $1.3 \mathrm{~m}$ Warsaw, Las Campanas, Chile \\
\hline OGLE-2007-BLG-006 & $\begin{array}{l}\text { OGLE, } 1.3 \text { m Warsaw, Las Campanas, Chile } \\
\mu \text { FUN, } 1.3 \text { m SMARTS, CTIO, Chile } \\
\mu \text { FUN, } 0.4 \text { m Auckland, New Zealand } \\
\mu \text { FUN, } 0.4 \text { m FCO, New Zealand }\end{array}$ \\
\hline $\begin{array}{l}\text { OGLE-2007-BLG-399/ } \\
\text { MOA-2007-BLG-334 }\end{array}$ & $\begin{array}{l}\text { OGLE, } 1.3 \mathrm{~m} \text { Warsaw, Las Campanas, Chile } \\
\text { MOA, } 2.0 \mathrm{~m} \text { Mt. John, New Zealand } \\
\mu \text { FUN, } 1.3 \mathrm{~m} \text { SMARTS, CTIO, Chile } \\
\text { PLANET, } 1.0 \mathrm{~m} \text { SAAO, South Africa }\end{array}$ \\
\hline $\begin{array}{l}\text { MOA-2011-BLG-104/ } \\
\text { OGLE-2011-BLG-0172 }\end{array}$ & $\begin{array}{l}\text { OGLE, } 1.3 \mathrm{~m} \text { Warsaw, Las Campanas, Chile } \\
\text { MOA, } 2.0 \mathrm{~m} \text { Mt. John, New Zealand } \\
\mu \mathrm{FUN}, 1.3 \mathrm{~m} \text { SMARTS, CTIO, Chile } \\
\mu \mathrm{FUN}, 0.4 \mathrm{~m} \text { Auckland, New Zealand } \\
\mu \mathrm{FUN}, 0.4 \mathrm{~m} \text { FCO, New Zealand } \\
\mu \mathrm{FUN}, 0.5 \mathrm{~m} \text { Wise, Israel } \\
\mu \mathrm{FUN}, 0.3 \mathrm{~m} \text { PEST, Australia } \\
\text { RoboNet, } 2.0 \mathrm{~m} \text { FTN, Hawaii } \\
\text { RoboNet, } 2.0 \mathrm{~m} \text { FTS, Australia }\end{array}$ \\
\hline MOA-2011-BLG-149 & $\begin{array}{l}\text { MOA, } 2.0 \mathrm{~m} \text { Mt. John, New Zealand } \\
\text { OGLE, } 1.3 \mathrm{~m} \text { Warsaw, Las Campanas, Chile } \\
\mu \text { FUN, } 1.3 \mathrm{~m} \text { SMARTS, CTIO, Chile } \\
\mu \text { FUN, } 0.4 \mathrm{~m} \text { Auckland, New Zealand } \\
\text { RoboNet, } 2.0 \mathrm{~m} \text { FTS, Australia } \\
\text { MiNDSTEp, } 1.54 \text { m Danish, La Silla, Chile }\end{array}$ \\
\hline $\begin{array}{l}\text { MOA-2011-BLG-278/ } \\
\text { OGLE-2011-BLG-012N }\end{array}$ & $\begin{array}{l}\text { MOA, } 2.0 \mathrm{~m} \text { Mt. John, New Zealand } \\
\text { OGLE, } 1.3 \mathrm{~m} \text { Warsaw, Las Campanas, Chile } \\
\mu \mathrm{FUN}, 1.3 \mathrm{~m} \text { SMARTS, CTIO, Chile } \\
\mu \text { FUN, } 0.4 \mathrm{~m} \text { Auckland, New Zealand } \\
\mu \mathrm{FUN}, 0.4 \mathrm{~m} \text { FCO, New Zealand } \\
\mu \mathrm{FUN}, 0.4 \mathrm{~m} \text { Kumeu, New Zealand } \\
\mu \text { FUN, } 1.0 \mathrm{~m} \text { Lemmon, Arizona } \\
\text { RoboNet, } 2.0 \mathrm{~m} \text { FTS, Australia } \\
\text { MiNDSTEp, } 1.54 \mathrm{~m} \text { Danish, La Silla, Chile }\end{array}$ \\
\hline
\end{tabular}

Notes. CTIO: Cerro Tololo Inter-American Observatory; FCO: Farm Cove Observatory; SAAO: South Africa Astronomical Observatory; PEST: Perth Extrasolar Survey Telescope; FTN: Faulkes North; FTS: Faulkes South.

field), and a sequential number assigned to the event. If an event is discovered independently by two different groups, they are named separately. Survey observations were conducted by two groups: the Optical Gravitational Lensing Experiment (OGLE; Udalski 2003) and the Microlensing Observation in Astrophysics (MOA; Bond et al. 2001; Sumi et al. 2003). Among seven events, five were additionally observed by follow-up observation groups, including $\mu$ FUN (Gould 2006), PLANET (Beaulieu et al. 2006), RoboNet (Tsapras et al. 2009), and MiNDSTEp (Dominik et al. 2010). In Table 2, we list the survey 


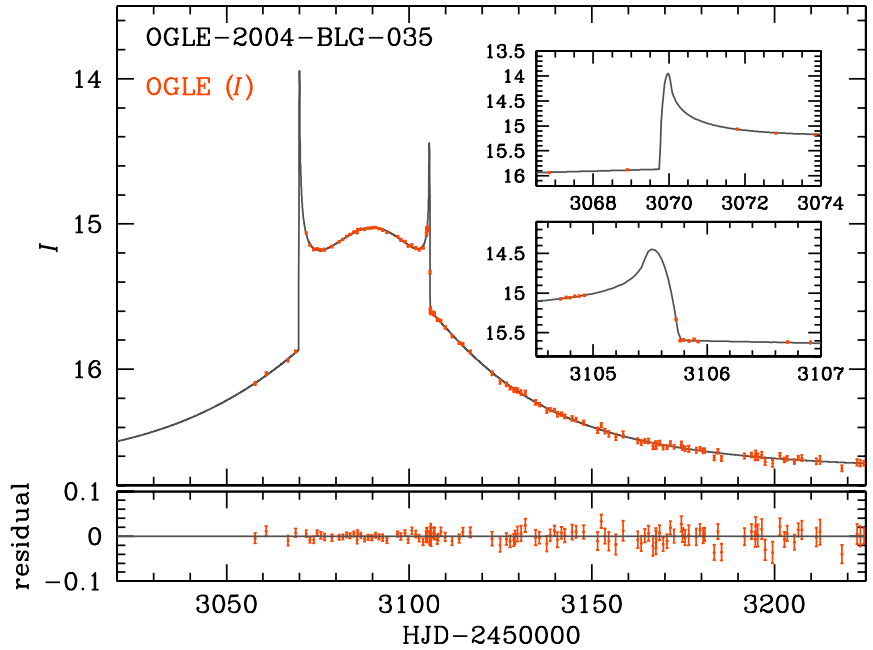

Figure 1. Light curve of OGLE-2004-BLG-035. The notation in the parentheses after the label of the observatory denotes the passband of observation. The insets show the enlargement of the light curve during caustic crossings and approaches. (A color version of this figure is available in the online journal.)

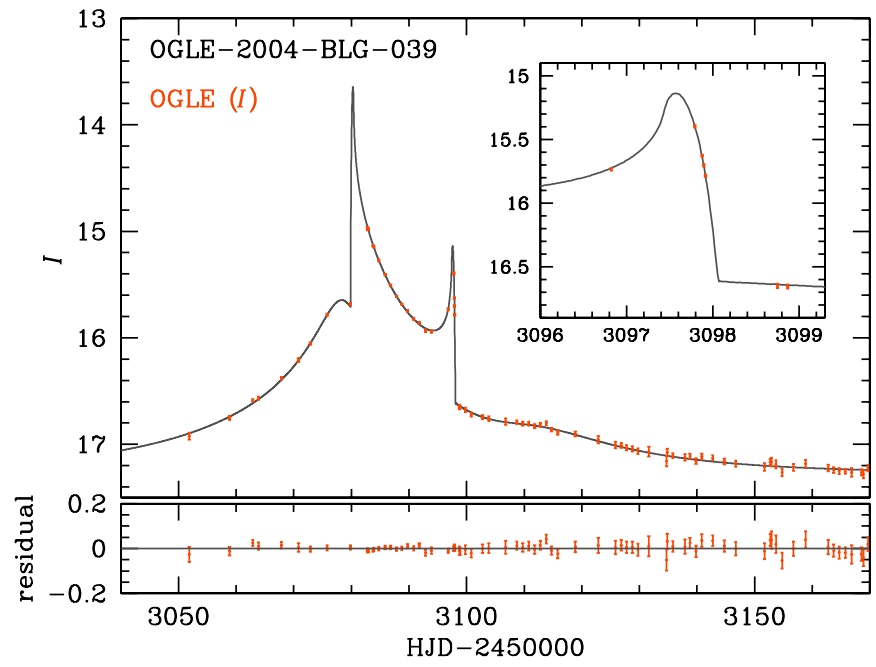

Figure 2. Light curve of OGLE-2004-BLG-039. Notations are same as in Figure 1.

(A color version of this figure is available in the online journal.)

and follow-up groups that participated in the observation of the individual events along with the telescopes and their locations.

Photometric reductions were carried out using photometry codes developed by the individual groups. The OGLE and MOA data were reduced by the photometry codes developed by Udalski (2003) and Bond et al. (2001), respectively, which are based on the Difference Image Analysis method (Alard \& Lupton 1998). The $\mu \mathrm{FUN}$ data were processed using a pipeline based on the DoPHOT software (Schechter et al. 1993). For PLANET and MiNDSTEp data, a pipeline based on the pySIS software (Albrow et al. 2009) is used. For the reduction of RoboNet data, the DanDIA pipeline (Bramich 2008) is used. Photometry errors of the individual data sets were rescaled so that $\chi^{2}$ per degree of freedom becomes unity for the data set of each observatory, where $\chi^{2}$ is estimated based on the bestfit model. We eliminate data points with large errors and those lying beyond $3 \sigma$ from the best-fit model to minimize their effect on modeling. In Figures 1-7, we present the light curves of the events.

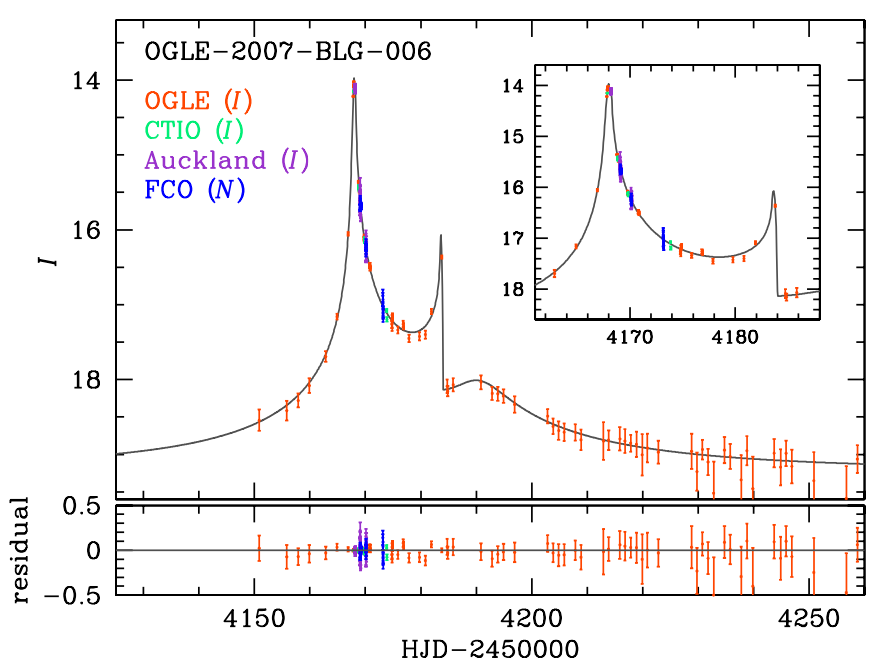

Figure 3. Light curve of OGLE-2007-BLG-006. Notations are same as in Figure 1. The notation for the observed passband " $N$ " denotes that no filter was used.

(A color version of this figure is available in the online journal.)

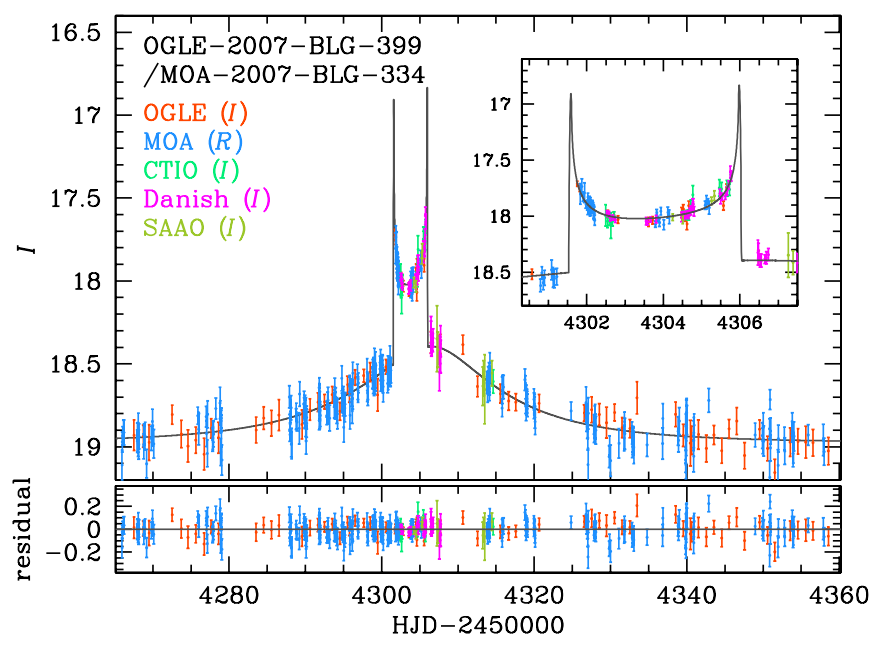

Figure 4. Light curve of OGLE-2007-BLG-399/MOA-2007-BLG-334. Notations are same as in Figure 1.

(A color version of this figure is available in the online journal.)

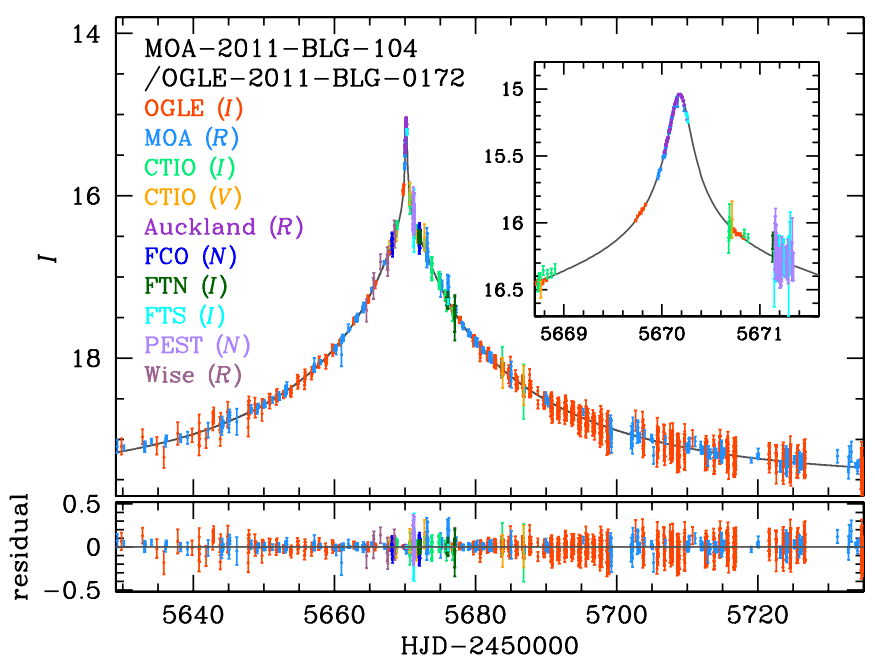

Figure 5. Light curve of MOA-2011-BLG-104/OGLE-2011-BLG-0172. Notations are same as in Figure 3.

(A color version of this figure is available in the online journal.) 


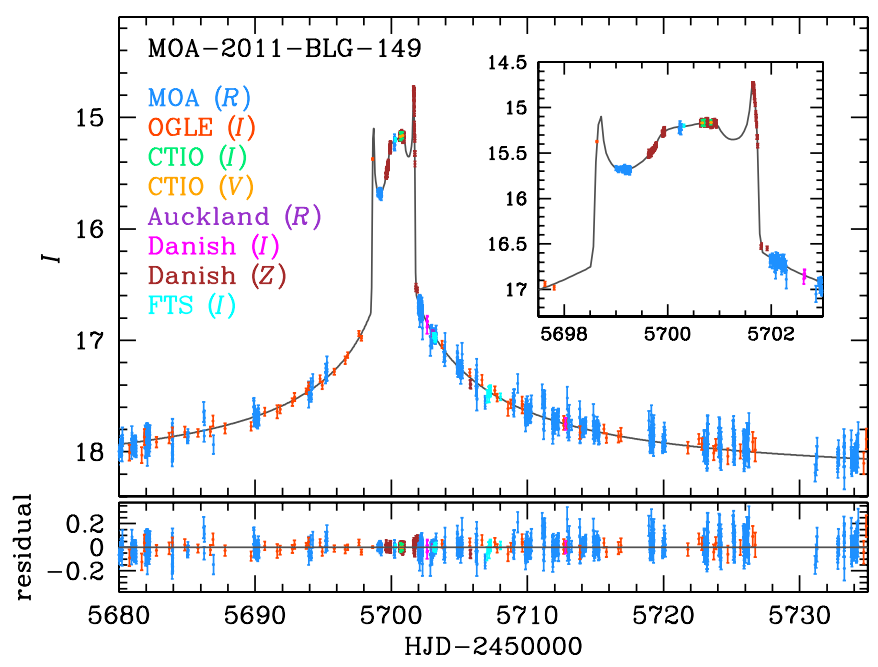

Figure 6. Light curve of MOA-2011-BLG-149. Notations are same as in Figure 1.

(A color version of this figure is available in the online journal.)

\section{MODELING}

In the analyses of each lensing light curve, we search for the solution of lensing parameters that yield the best fit to the observed light curve. The basic structure of a binary-lensing light curve is described by seven parameters. The first three parameters $t_{0}, u_{0}$, and $t_{\mathrm{E}}$ are identical to those of a single-lensing event and specify the source motion with respect to the lens. The next three parameters describe the binary-lens system, including the projected separation between lens components in units of the Einstein radius, $s$, the mass ratio between the lens components, $q$, and the angle between source trajectory and the binary axis, $\alpha$. The final parameter is the source radius, $\theta_{\star}$, normalized to the Einstein radius, $\rho_{\star}=\theta_{\star} / \theta_{\mathrm{E}}$ (normalized source radius). The normalized source radius is needed to account for dilution of the lensing magnification caused by the finite size of the source. The finite source effect is important when the source is located at a position where the magnification varies very rapidly such as near the caustic, and thus different parts of the source star are magnified by different amounts. In addition to these basic binary-lensing parameters, it is often needed to consider the parallax effect for a fraction of events with long timescales. To describe the lens parallax effect, it is required to include two additional parameters, $\pi_{\mathrm{E}, N}$ and $\pi_{\mathrm{E}, E}$, which represent the two components of the lens parallax vector $\boldsymbol{\pi}_{\mathrm{E}}$ projected on the sky along the north and east equatorial coordinates, respectively. The size of the lens parallax vector corresponds to the ratio of Earth's orbit to the Einstein radius projected on the observer's plane, i.e., $\pi_{\mathrm{E}}=\mathrm{AU} /\left[r_{\mathrm{E}} D_{\mathrm{S}} /\left(D_{\mathrm{S}}-D_{\mathrm{L}}\right)\right]$, where $r_{\mathrm{E}}=D_{\mathrm{L}} \theta_{\mathrm{E}}$ is the physical size of the Einstein radius. See Equation (4).

In the analysis, we search for the best-fit solution in two steps. In the first step, a grid search is conducted for a subset of parameters, while other parameters are optimized by using a downhill approach (Dong et al. 2006). By inspecting the $\chi^{2}$ distribution in the grid parameter space, we identify local minima. In the second step, we investigate the individual local minima and further refine the solutions by allowing all parameters to vary. Once the solutions corresponding to the local minima are found, we choose the best-fit solution by comparing the $\chi^{2}$ values of the individual local minima. In this process, we also identify possible degenerate solutions resulting from various causes. We set $s, q$, and $\alpha$ as grid parameters

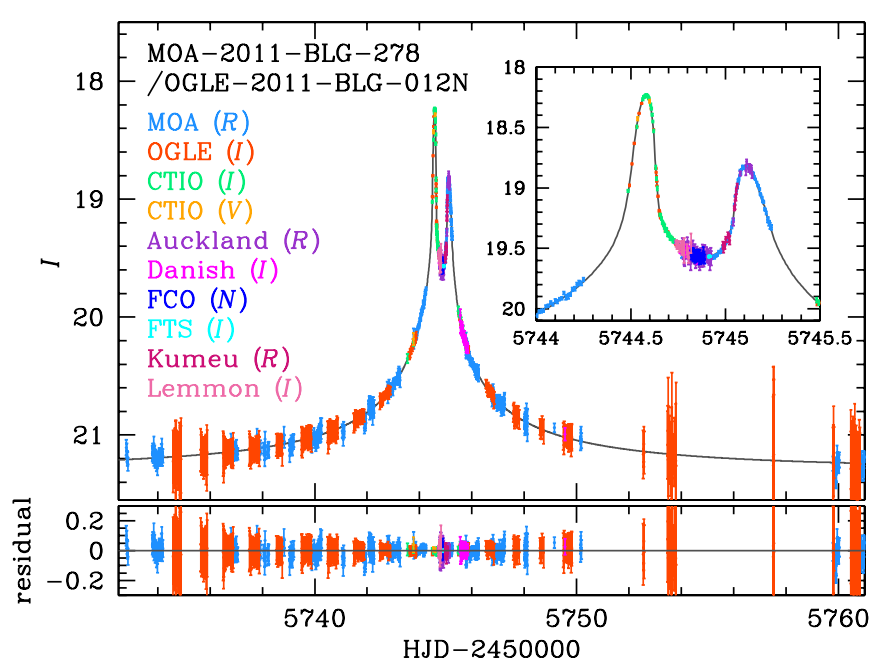

Figure 7. Light curve of MOA-2011-BLG-278/OGLE-2011-BLG-012N. Notations are same as in Figure 3.

(A color version of this figure is available in the online journal.)

because these parameters are related to light-curve features in a complex way such that a small change in the values of the parameters can lead to dramatic changes in the resulting light curve. For the $\chi^{2}$ minimization in the downhill approach, we use the Markov Chain Monte Carlo (MCMC) method. Once the solution of the parameters is found, we estimate the uncertainties on the individual parameters based on the distributions of the parameters obtained from the MCMC chain of solutions.

We use the inverse ray-shooting technique (Schneider \& Weiß 1986; Kayser et al. 1986; Wambsganss 1997) to compute magnifications of events affected by the finite source effect. In this numerical method, rays are uniformly shot from the image plane, bent by the lens equation, and land on the source plane. Then, the finite magnification is computed as the ratio of the number density of rays on the source plane to that on the image plane. The lens equation for a binary lens, which describes the relation between the positions of a ray on the image and source planes, is represented by

$$
\zeta=z-\frac{m_{1}}{\bar{z}-\bar{z}_{\mathrm{L}, 1}}-\frac{m_{2}}{\bar{z}-\bar{z}_{\mathrm{L}, 2}},
$$

where $m_{1}=1 /(1+q)$ and $m_{2}=q m_{1}$ are the mass fractions of the binary-lens components; $\zeta=\xi+i \eta, z=x+i y$, and $z_{\mathrm{L}}=x_{\mathrm{L}}+i y_{\mathrm{L}}$ represent the complex notations of the source, image, and lens locations, respectively; and $\bar{z}$ denotes the complex conjugate of $z$. The ray-shooting method requires heavy computation. We thus minimize computation time by applying the ray-shooting method only when the source crosses or approaches very close to caustics and utilizing the semianalytic hexadecapole approximation (Gould 2008; Pejcha \& Heyrovský 2009) in the adjacent area.

In computing finite magnifications, we consider the variation of the magnification caused by the limb darkening of the source star surface for events with well-resolved features involving caustics. In our model, the surface brightness profile is described by

$$
S_{\lambda}=\frac{F_{\lambda}}{\pi \theta_{\star}^{2}}\left[1-\Gamma_{\lambda}\left(1-\frac{3}{2} \cos \psi\right)\right],
$$

where $\Gamma_{\lambda}$ is the linear limb-darkening coefficient, $F_{\lambda}$ is the source star flux, and $\psi$ is the angle between the normal to the source star's surface and the line of sight toward the star. We 
Table 3

Limb-darkening Coefficients and Source Information

\begin{tabular}{lccc}
\hline \hline Quantity & $\begin{array}{c}\text { MOA-2011-BLG-104/ } \\
\text { OGLE-2011-BLG-0172 }\end{array}$ & MOA-2011-BLG-149 & $\begin{array}{c}\text { MOA-2011-BLG-278/ } \\
\text { OGLE-2011-BLG-012N }\end{array}$ \\
\hline$\Gamma_{V}$ & 0.51 & 0.73 & 0.59 \\
$\Gamma_{R}$ & 0.43 & 0.64 & 0.51 \\
$\Gamma_{I}$ & 0.36 & 0.53 & 0.43 \\
$(V-I)_{0}$ & 0.62 & 1.64 & 0.85 \\
Source type & $\mathrm{FV}$ & $\mathrm{KV}$ & $\mathrm{GV}$ \\
$T_{\text {eff }}(\mathrm{K})$ & 6650 & 4410 & 5790 \\
$v_{\text {turb }}\left(\mathrm{km} \mathrm{s}^{-1}\right)$ & 2 & 2 & 2 \\
$\log g\left(\mathrm{~cm} \mathrm{~s}^{-2}\right)$ & 4.5 & 4.5 & 4.5 \\
\hline
\end{tabular}

Table 4

Model Parameters

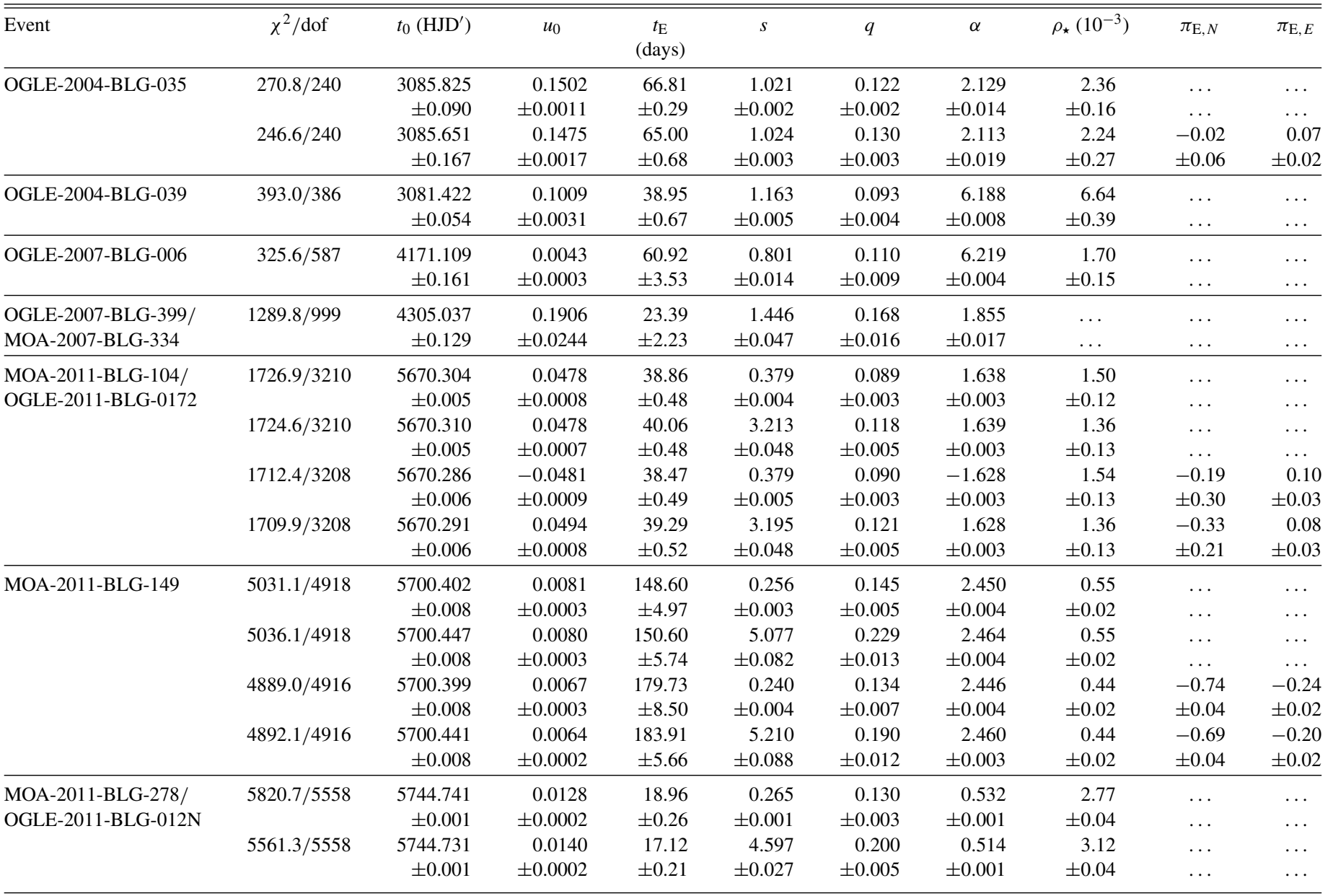

Note. $\mathrm{HJD}^{\prime}=\mathrm{HJD}-2,450,000$.

set the limb-darkening coefficients corresponding to the source type that is determined based on the color and magnitude of the source. In Table 3, we present the adopted limb-darkening coefficients, corresponding source types, and the measured dereddened color, along with the assumed values of the effective temperature, $T_{\text {eff }}$, the surface turbulence velocity, $v_{\text {turb }}$, and the surface gravity, $\log g$.

For all seven events, we conduct modeling based on the seven basic binary-lensing parameters. We carry out additional modeling considering the parallax effect for events with Einstein timescales $t_{\mathrm{E}}>30$ days. When the parallax effect is considered, we separately investigate solutions with impact parameters $u_{0}>0$ and $u_{0}<0$. This is because the lensing light curves resulting from the source trajectories with $+u_{0}$ and $-u_{0}$ are different due to the asymmetry of the trajectory caused by the parallax effect, while the light curves are identical when the trajectories are straight lines due to the symmetry of the magnification pattern with respect to the binary axis.

\section{RESULTS}

In Table 4, we present the solutions of the parameters determined from modeling for the individual events. For events where the additional parallax modeling is conducted, we also list the parallax solution. In addition, we present the solutions of local minima if they exist. The model light curves resulting from 

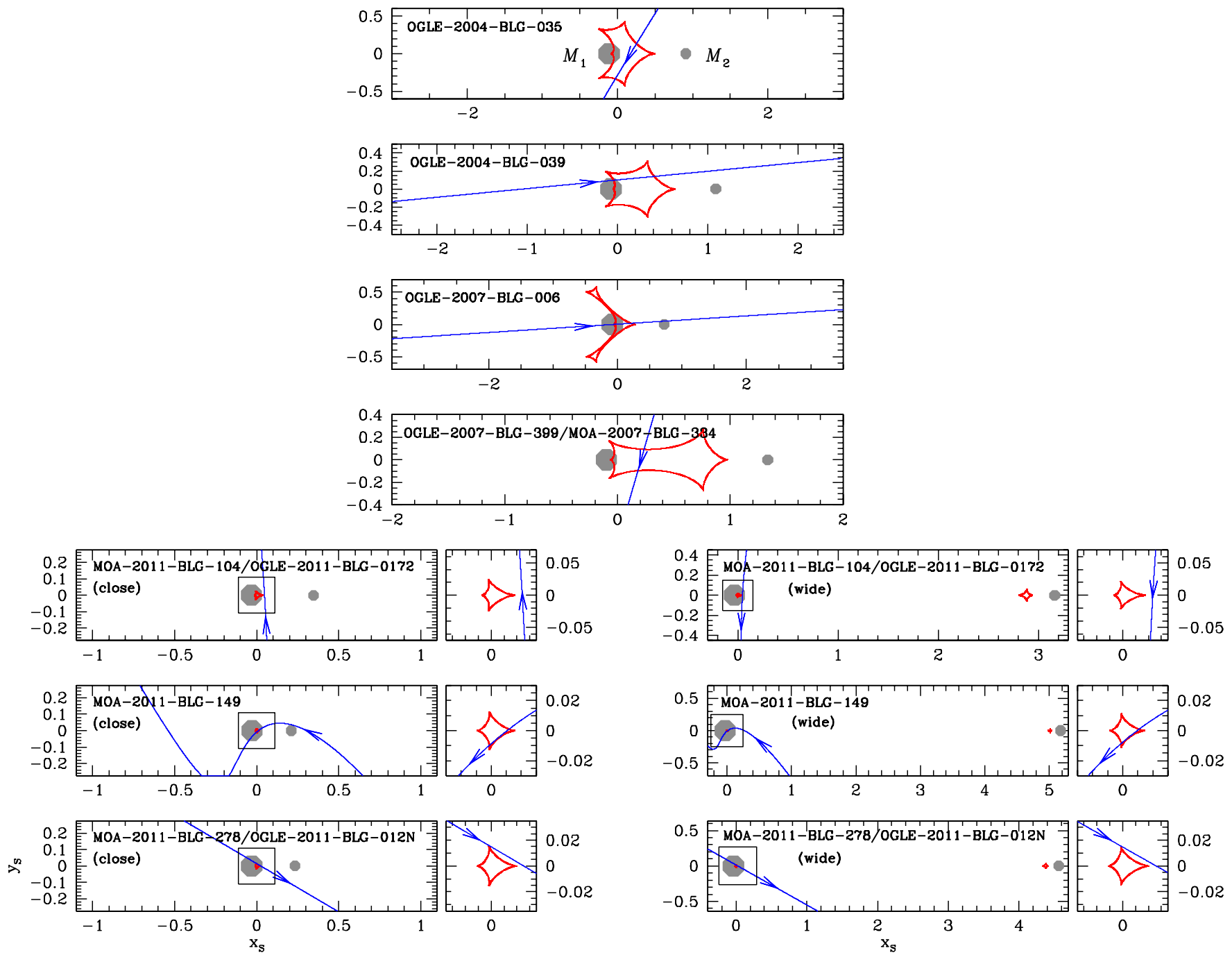

Figure 8. Geometry of the binary-lens systems for the light curves presented in Figures 1-7. In each panel, the two dots (marked by $M_{1}$ and $M_{2}$ ) represent the locations of the binary-lens components, where the bigger $\left(M_{1}\right)$ and smaller $\left(M_{2}\right)$ dots represent the higher and lower mass binary components, respectively. The figure composed of concave curves is the caustic. The line with an arrow represents the source trajectory. For the three events MOA-2011-BLG-104/OGLE-2011-BLG-0172, MOA-2011-BLG-149, and MOA-2011-BLG-278/OGLE-2011-BLG-012N, we present the geometry of two solutions caused by the close/wide degeneracy. To better show the caustic-involved perturbation region for each of the three events with degenerate close and wide solutions, we present the enlargement of the perturbation region (enclosed by a box) in a separate panel on the right side of each main panel.

(A color version of this figure is available in the online journal.)

the best-fit solutions of the individual events are presented on the top of the observed data points in Figures 1-7. In Figure 8, we also present the geometry of the lens system corresponding to the best-fit solution of each event. Based on the best-fit solutions, it is found that the mass ratios of the analyzed events are in the range $0.09<q<0.20$, indicating that the lower mass companions of the lenses are strong brown dwarf candidates.

For three out of the seven events, it is found that there exist local minima other than the best-fit solution. The events with degenerate solutions include MOA-2011-BLG-104/OGLE-2011BLG-0172, MOA-2011-BLG-149, and MOA-2011-BLG-278/ OGLE-2011-BLG-012N. For all of these events, the degeneracy is caused by the symmetry of the lens equations between the close $(s<1)$ and wide $(s>1)$ binaries, which is known as the "close/wide binary degeneracy" (Dominik 1999). For MOA-2011-BLG-104/OGLE-2011-BLG-0172 and MOA2011-BLG-149, the degeneracy is very severe, with $\Delta \chi^{2}=2.5$ and 3.1, respectively. For MOA-2011-BLG-278/OGLE-2011BLG-012N, the degeneracy is less severe and the wide binary solution is preferred over the close binary solution with
$\Delta \chi^{2}=259.4$. We note that the mass ratios of the pair of close and wide binary solutions are similar to each other although the binary separations are very different. Therefore, the degeneracy does not affect the brown dwarf candidacy of the lens.

For three events, it is possible to measure the lens mass by simultaneously measuring the lens parallax and the Einstein radius. With these values, the lens mass and distance are determined by

$$
M=\frac{\theta_{\mathrm{E}}}{\kappa \pi_{\mathrm{E}}}
$$

and

$$
D_{\mathrm{L}}=\frac{\mathrm{AU}}{\pi_{\mathrm{E}} \theta_{\mathrm{E}}+\pi_{\mathrm{S}}},
$$

respectively. Here $\pi_{\mathrm{S}}=\mathrm{AU} / D_{\mathrm{S}}$ represents the parallax of the source star.

For MOA-2011-BLG-104/OGLE-2011-BLG-0172, the value of the lens parallax measured from modeling is $\pi_{\mathrm{E}}=$ $\left(\pi_{\mathrm{E}, N}{ }^{2}+\pi_{\mathrm{E}, E^{2}}\right)^{1 / 2}=0.34 \pm 0.21$. The Einstein radius is measured based on the normalized source radius determined from 

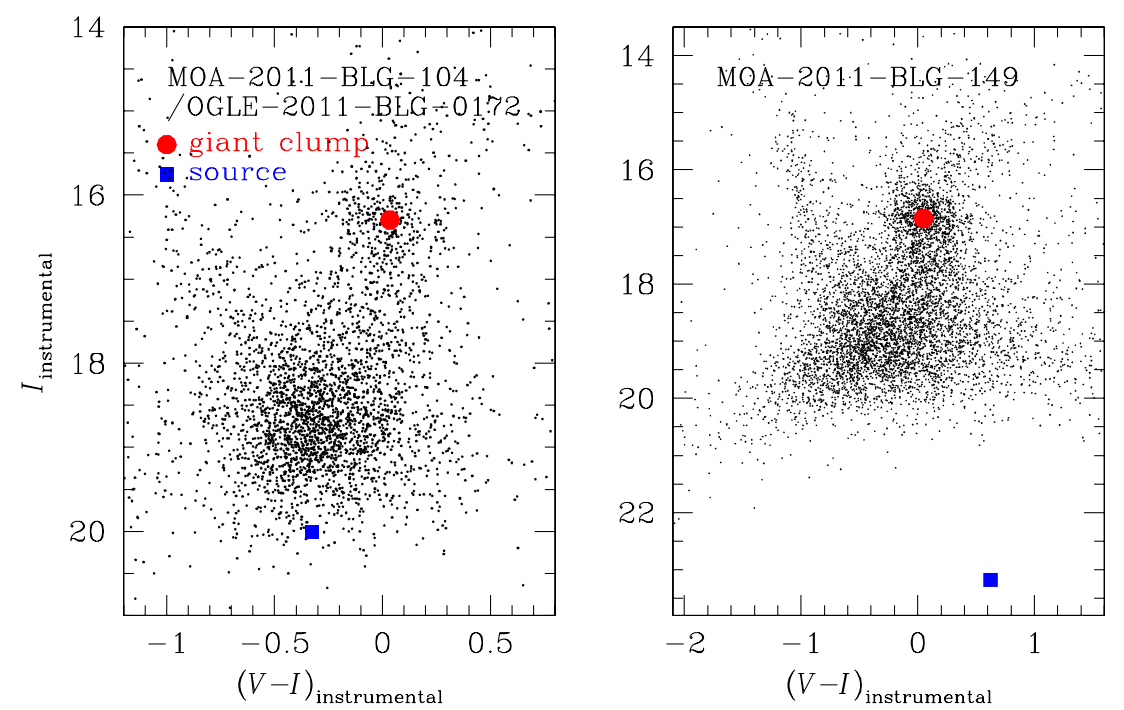

Figure 9. Color-magnitude diagram of stars in the field of MOA-2011-BLG-104/OGLE-2011-BLG-0172 and MOA-2011-BLG-149 with the locations of the lensed stars and giant clumps marked as blue squares and red circles, respectively.

(A color version of this figure is available in the online journal.)

modeling combined with the information on the angular source size using $\theta_{\mathrm{E}}=\theta_{\star} / \rho_{\star}$. The angular source size is assessed from the de-reddened color and brightness of the source star, which are estimated based on the source brightness measured in $V$ and $I$ passbands and calibrated by the relative position of the source on the color-magnitude diagram with respect to a reference (Yoo et al. 2004). We use the centroid of the bulge giant clump as the reference because its de-reddened brightness of $I_{0, \mathrm{c}}=14.45$ at the Galactocentric distance (D. M. Nataf \& A. Gould 2012, in preparation $)^{80}$ and the color of $(V-I)_{0, c}=1.06$ (Bensby et al. 2011) are known. We measure the de-reddened color and brightness of the source star to be $(V-I, I)_{0, \mathrm{~s}}=(0.70,18.15)$. Figure 9 shows the locations of the source star and the centroid of the giant clump in the color-magnitude diagram that is constructed based on the CTIO data. After the initial photometric characterization of the source, we learned that spectroscopic observation is conducted when the source was highly magnified (T. Bensby 2012, private communication). From this spectroscopic observation, the de-reddened color of the source star is estimated as $(V-I)_{0, \mathrm{~s}}=0.62$, which is adopted for our analysis. Once the de-reddened $V-I$ color of the source star is measured, it is translated into $V-K$ color by using the $V-I$ versus $V-K$ relations of Bessell \& Brett (1988) and then the source angular radius is estimated by using the relation between the $V-K$ color and the angular radius given by Kervella et al. (2004). The measured angular source radius and the Einstein radius are $\theta_{\star}=(0.68 \pm 0.06) \mu$ as and $\theta_{\mathrm{E}}=(0.54 \pm 0.07)$ mas, respectively. With the measured Einstein radius, the mass and distance to the lens are estimated as $M=(0.20 \pm 0.12) M_{\odot}$ and $D_{\mathrm{L}}=(3.29 \pm 1.20) \mathrm{kpc}$, respectively. Then, the corresponding masses of the primary and the companion are $M_{1}=M /(1+q)=(0.18 \pm 0.11) M_{\odot}$ and $M_{2}=q M_{1}=(0.02 \pm 0.01) M_{\odot}$, respectively. Therefore, the binary lens is composed of a low-mass $\mathrm{M}$ dwarf and a brown dwarf.

For MOA-2011-BLG-149, the lens parallax is also measured with $\pi_{\mathrm{E}}=0.78 \pm 0.04$. For this event, however, multi-band ( $V$ and $I$ ) observations taken at a single observatory (CTIO) covered

\footnotetext{
${ }^{80}$ For other Galactic bulge fields with Galactic coordinates $(l, b)$, the $I$-band brightness is given by $I_{0, \mathrm{c}}=14.45-5 \log (\cos l+\sin l \cos \phi / \sin \phi)$, where $\phi \sim 45^{\circ}$ is the orientation angle of the Galactic bulge with respect to the line
} of sight. only near the high magnification peak of the light curve. As a result, the source brightness in both bands cannot be estimated by the usual method based on modeling. Fortunately, there exists no significant blending for this event, and thus the color measured at the peak can be approximated as the source color. From the source brightness and magnification at the peak, we also estimate the baseline source brightness. The source location estimated in this way is plotted on the color-magnitude diagram presented in Figure 9. Once the source location is determined, the de-reddened color and brightness are determined by the usual way of using the clump centroid. The measured values are $(V-I, I)_{0, \mathrm{~s}}=(1.64,20.8)$, which correspond to those of a Galactic bulge K-type dwarf with an angular radius of $\theta_{\star}=(0.46 \pm 0.04) \mu$ as. Then, the estimated Einstein radius is $\theta_{\mathrm{E}}=(1.04 \pm 0.10)$ mas and the resulting lens mass and distance are $M=(0.16 \pm 0.02) M_{\odot}$ and $D_{\mathrm{L}}=(1.07 \pm 0.10) \mathrm{kpc}$, respectively. The masses of the individual lens components are $M_{1}=(0.14 \pm 0.02) M_{\odot}$ and $M_{2}=(0.019 \pm 0.002) M_{\odot}$, respectively. Therefore, the lens system is also composed of a low-mass $\mathrm{M}$ dwarf and a brown dwarf.

For another parallax event OGLE-2004-BLG-035, no multiband observation is conducted. However, the source star of the event is very bright, implying that the source flux is not much affected by blended light. Under this approximation, we measure the source color in the OGLE catalog. Based on this approximate color combined with the giant clump location, we estimate the de-reddened color and brightness of the source star as $(V-I, I)_{0, \mathrm{~s}}=(1.19,15.83)$, which correspond to the values of a low-luminosity giant star with a source radius of $\theta_{\star}=(3.72 \pm 0.32) \mu$ as. Considering that there exists a non-zero blended flux fraction $F_{\mathrm{b}} / F_{\text {tot }} \sim 20 \%$, we decrease the source radius by $\sim\left(F_{\mathrm{b}} / F_{\text {tot }}\right) / 2=10 \%$. The Einstein radius obtained is $\theta_{\mathrm{E}}=(1.40 \pm 0.21)$ mas. Then, the mass of the lens system is $M=(2.48 \pm 0.87) M_{\odot}$, and thus the mass of the companion is $M_{2}=(0.29 \pm 0.10) M_{\odot}$. The companion mass is greater than the upper mass limit of brown dwarfs of $0.08 M_{\odot}$.

\section{CONCLUSION}

We searched for candidate binaries with brown dwarf companions by analyzing binary microlensing events 
discovered during the 2004-2011 observing seasons. Under the low mass ratio criterion of $q<0.2$, we found seven candidate events: OGLE-2004-BLG-035, OGLE-2004-BLG039, OGLE-2007-BLG-006, OGLE-2007-BLG-399/ MOA-2007-BLG-334, MOA-2011-BLG-104/OGLE-2011BLG-0172, MOA-2011-BLG-149, and MOA-201-BLG-278/ OGLE-2011-BLG-012N. Among them, we confirmed that the companions of MOA-2011-BLG-104/OGLE-2011-BLG-0172 and MOA-2011-BLG-149 were brown dwarfs by measuring the lens masses.

The number of microlensing brown dwarfs is expected to increase with the improvements in lensing surveys and analysis techniques. With the adoption of a new wide-field camera, the number of lensing events detected by the OGLE survey is increased by a factor of more than two. In addition, a new survey based on a network of three telescopes located at three different locations in the Southern Hemisphere (Korea Microlensing Telescope Network) is planned to operate from the 2014 season. Along with the improvements on the observational side, the analysis technique has greatly improved in recent years, enabling prompt and precise analysis of a large number of anomalous events. With this improvement, the number of microlensing brown dwarfs will rapidly increase, making microlensing one of the major methods in the discovery of brown dwarfs.

Work by C.H. was supported by the Creative Research Initiative Program (2009-0081561) of the National Research Foundation of Korea. The OGLE project has received funding from the European Research Council under the European Community's Seventh Framework Programme (FP7/2007-2013)/ERC grant agreement no. 246678. The MOA experiment was supported by grants JSPS22403003 and JSPS23340064. T.S. was supported by the grant JSPS23340044. Y. Muraki acknowledges support from JSPS grants JSPS23540339 and JSPS19340058. The MiNDSTEp monitoring campaign is powered by ARTEMiS (Automated Terrestrial Exoplanet Microlensing Search; Dominik et al. 2008). M.H. acknowledges support by the German Research Foundation (DFG). D.R. (boursier FRIA), F.F. (boursier ARC), and J. Surdej acknowledge support from the Communauté française de Belgique Actions de recherche concertées - Académie universitaire Wallonie-Europe. K.A., D.M.B., M.D., K.H., M.H., C.L., C.S., R.A.S., and Y.T. are grateful to Qatar National Research Fund (QNRF), member of Qatar Foundation, for support by grant NPRP 09-476-1078. C.S. received funding from the European Union Seventh Framework Programme (FPT/2007-2013) under grant agreement no. 268421. This work is based in part on data collected by MiNDSTEp with the Danish $1.54 \mathrm{~m}$ telescope at the ESO La Silla Observatory. The Danish $1.54 \mathrm{~m}$ telescope is operated based on a grant from the Danish Natural Science Foundation (FNU). A. Gould and B. S. Gaudi acknowledge support from NSF AST-1103471. B. S. Gaudi, A. Gould, and R. W. Pogge acknowledge support from NASA grant NNG04GL51G. Work by J. C. Yee is supported by a National Science Foundation Graduate Research Fellowship under grant no. 2009068160. S. Dong's research was performed under contract with the California Institute of Technology (Caltech) funded by NASA through the Sagan Fellowship Program. Research by T.C.H. was carried out under the KRCF Young Scientist Research Fellowship Program. T.C.H. and C.U.L. acknowledge the support of Korea Astronomy and Space Science Institute (KASI) grant 2012-1-410-02. I.A.B. and P.C.M.Y. acknowledge the support by the Marsden Fund of New Zealand.

\section{REFERENCES}

Alard, C., \& Lupton, R. H. 1998, ApJ, 503, 325

Albrow, M. D., Horne, K., Bramich, D. M., et al. 2009, MNRAS, 397, 2099

Beaulieu, J.-P., Bennett, D. P., Fouqué, P., et al. 2006, Nature, 439, 437

Bensby, T., Adén, D., Meléndez, J., et al. 2011, A\&A, 533, 134

Bessell, M. S., \& Brett, J. M. 1988, PASP, 100, 1134

Bond, I. A., Abe, F., Dodd, R. J., et al. 2001, MNRAS, 327, 868

Bozza, V., Dominik, M., Rattenbury, N. J., et al. 2012, MNRAS, 424, 902

Bramich, D. M. 2008, MNRAS, 386, L77

Choi, J.-Y., Shin, I.-G., Park, S.-Y., et al. 2012, ApJ, 751, 41

Dominik, M. 1999, A\&A, 349, 108

Dominik, M., Horne, K., Allan, A., et al. 2008, Astron. Nachr., 329, 248

Dominik, M., Jørgensen, U. G., Rattenbury, N. J., et al. 2010, Astron. Nachr., 331,671

Dong, S., DePoy, D. L., Gaudi, B. S., et al. 2006, ApJ, 642, 842

Gould, A. 1992, ApJ, 392, 442

Gould, A. 1994, ApJ, 421, L71

Gould, A. 2008, ApJ, 681, 1593

Gould, A., Udalski, A., An, D., et al. 2006, ApJ, 644, L37

Gould, A., Udalski, A., Monard, B., et al. 2009, ApJ, 698, L147

Graff, D., \& Freese, K. 1996, ApJ, 456, L49

Han, C., \& Gould, A. 2003, ApJ, 592, 172

Jaroszyński, M., \& Skowron, J. 2008, Acta Astron., 53, 345

Jaroszyński, M., Skowron, J., Udalski, A., et al. 2006, Acta Astron., 56, 307

Jaroszyński, M., Skowron, J., Udalski, A., et al. 2010, Acta Astron., 60, 197

Kayser, R., Refsdal, S., \& Stabell, R. 1986, A\&A, 166, 36

Kervella, P., Thévenin, F., Di Folco, E., \& Ségransan, D. 2004, A\&A, 426, 297

Kulkarni, S. R. 1997, Science, 276, 1350

Kumar, S. S. 1969, Low-luminosity Stars (London: Gordon and Breach)

Mainzer, A., Cushing, M. C., Skrutskie, M., et al. 2011, ApJ, 726, 30

Mao, S., \& Paczyński, B. 1991, ApJ, 374, L37

Najita, J., Tiede, G., \& Carr, S. 2000, ApJ, 541, 977

Nakajima, T., Oppenheimer, B. R., Kulkarni, S. R., et al. 1995, Nature, 378,463

Nemiroff, R. J., \& Wickramasinghe, W. A. D. T. 1994, ApJ, 424, L21

Paczyński, B. 1986, ApJ, 304, 1

Pejcha, O., \& Heyrovský, D. 2009, ApJ, 690, 1772

Rebolo, R., Zapatero Osorio, M. R., \& Martín, E. L. 1995, Nature, 377, 129

Ryu, Y.-H., Han, C., Hwang, K.-H., et al. 2010, ApJ, 723, 81

Schechter, P. L., Mateo, M., \& Saha, A. 1993, PASP, 105, 1342

Schneider, P., \& Weiß, A. 1986, A\&A, 164, 237

Schuh, S. L., Handler, G., Drechsel, H., et al. 2003, A\&A, 410, 649

Skowron, J., Jaroszyński, M., Udalski, A., et al. 2007, Acta Astron., 57, 281

Smith, C., Mao, S., \& Wozńiak, P. 2003, ApJ, 585, L65

Sumi, T., Abe, F., Bond, I. A., et al. 2003, ApJ, 591, 204

Tisserand, P., Le Guillou, L., Afonso, C., et al. 2007, A\&A, 469, 387

Tsapras, Y., Street, R., Horne, K., et al. 2009, Astron. Nachr., 330, 4

Udalski, A. 2003, Acta Astron., 53, 291

Wambsganss, J. 1997, MNRAS, 284, 172

Witt, H., \& Mao, S. 1994, ApJ, 430, 505

Yoo, J., DePoy, D. L., Gal-Yam, A., et al. 2004, ApJ, 603, 139 